\title{
Extremely Strong Boundary Points and Real-linear Isometries
}

\author{
Arya JAMSHIDI and Fereshteh SADY
}

Tarbiat Modares University

(Communicated by M. Hara)

\begin{abstract}
Let $X, Y$ be locally compact Hausdorff spaces, $A$ be a complex subspace of $C_{0}(X)$ and $T: A \rightarrow$ $C_{0}(Y)$ be a real-linear isometry, whose range is not assumed to be a complex subspace of $C_{0}(Y)$. In this paper, using the set $\Theta(A)$ and $\tau(A)$ consisting of all extremely strong boundary points and strong boundary points of $A$, respectively we introduce appropriate subsets $Y_{0}$ and $Y_{1}$ of $Y$ and give a description of $T$ on these sets. More precisely, we show that there exist continuous functions $\Phi: Y_{0} \rightarrow \Theta(A), \alpha: Y_{0} \rightarrow[-1,1]$ and $w: Y_{0} \rightarrow \mathbb{T}$, where $\mathbb{T}$ is the unit circle, such that
\end{abstract}

$$
T f(y)=w(y) \cdot(\operatorname{Re}(f(\Phi(y)))+\alpha(y) i \operatorname{Im}(f(\Phi(y)))
$$

for all $f \in A$ and $y \in Y_{0}$. The result is improved in the case where either

i) $T(A)$ is a complex subspace of $C_{0}(Y)$ and $\Theta(A)=\operatorname{ch}(A)$, where $\operatorname{ch}(A)$ is the Choquet boundary of $A$ or

ii) $T(A)$ satisfies a certain separating property.

In the first case we show that there exists a clopen subset $K$ of $Y_{0}$ such that

$$
(T f)(y)=w(y) \begin{cases}\frac{f(\Phi(y))}{f(\Phi(y))} & y \in K, \\ y \notin K,\end{cases}
$$

for each $f \in A$ and $y \in Y_{0}$. In the second case we obtain similar results for $\tau(A) \cap \operatorname{ch}(A)$ and $Y_{1}$ instead of $\Theta(A)$ and $Y_{0}$.

\section{Introduction}

For a compact (resp. locally compact) Hausdorff space $X$, let $C(X)$ (resp. $\left.C_{0}(X)\right)$ denote the Banach space of all complex-valued continuous functions on $X$ (resp. complex-valued continuous functions on $X$ vanishing at infinity) endowed with the supremum norm $\|\cdot\|_{\infty}$. By the classical Banach-Stone Theorem, every linear isometry $T$ from $C(X)$ onto $C(Y)$, where $X, Y$ are compact Hausdorff spaces, is a weighted composition operator of the form $T f(y)=$ $h(y) f(\Phi(y))$ for all $y \in Y$ and $f \in C(X)$, where $h \in C(Y)$ is unimodular and $\Phi: Y \rightarrow X$ is a homeomorphism. Linear (not necessarily surjective) isometries between certain subspaces of continuous functions have been studied extensively. For instance, in [12] Novinger gave a description of a linear isometry $T: A \rightarrow C_{0}(Y)$ on the Choquet boundary of its range, where

Received May 19, 2014; revised December 10, 2014

2010 Mathematics Subject Classification: 47B04 (Primary), 47B48 (Secondary)

Key words and phrases: real-linear isometry, strong boundary point, extremely strong boundary point, uniform algebra 
$A$ is a subspace of $C(X)$, for some compact Hausdorff space $X$, which contains the constant and separates the points of $X$ and $Y$ is a locally compact Hausdorff space. In [1], linear isometries between strongly separating subspaces of $C_{0}(X)$, for locally compact Hausdorff space $X$, have been studied and the general form of such isometries have been characterized. Here a subspace $A$ of $C_{0}(X)$ is strongly separating if for distinct points $x, y \in X$ there exists $f \in A$ with $|f(x)| \neq|f(y)|$. A well-known generalization of the Banach-Stone theorem given by de Leeuw, Rudin and Wermer in [9] states that a linear surjective isometry between closed separating unital subalgebras of continuous functions on a compact Hausdorff space $X$ (called uniform algebras on $X$ ) is a multiple by a unimodular continuous function of an algebra isomorphism. For a uniform algebra $A$ on a compact Hausdorff space $X$ and strictly positive function $f \in C(X)$, linear isometries and $A$-module isometries between closed subspaces of the form $A f$ have been discussed in [2].

Since, by the Mazur-Ulam theorem, every surjective isometry between normed spaces is real-linear up to a translation, it may be interesting to consider real-linear isometries between various normed spaces, particularly, on subspaces of continuous functions. For instance in [5] Ellis considered real-linear isometries in the case where the underlying topological spaces are the Shilov boundaries of subspaces under consideration. In [16] Tonev and Yates proved that, under some additional assumptions, a surjective map $T: A \rightarrow B$ between uniform algebras $A$ and $B$ on compact Hausdorff spaces $X$ and $Y$, satisfying $\|T(f)+T(g)\|_{\infty}=$ $\|f+g\|_{\infty}$, for all $f, g \in A$, (which means that $T$ is a real-linear isometry) is an isometric algebra isomorphism which induces a homeomorphism between the Choquet boundaries of $A$ and $B$. In [6] Hatori and et al. considered a similar problem for maps between (unital) semisimple commutative Banach algebras preserving additively the spectral radius of algebra elements. For locally compact Hausdorff spaces $X, Y$, real-linear surjective isometries $T$ : $A \rightarrow B$ between strongly separating subalgebras $A$ and $B$ of $C_{0}(X)$ and $C_{0}(Y)$ were studied by Miura in [11] and it was shown that there exist a continuous function $\kappa$ from the Choquet boundary $\operatorname{ch}(B)$ of $B$ to the unit circle, a clopen subset $K$ of $\operatorname{ch}(B)$ and a homeomorphism $\varphi: \operatorname{ch}(B) \rightarrow \operatorname{ch}(A)$ such that $T(f)=\kappa(f \circ \varphi)$ on $K$ and $T(f)=\kappa(\overline{f \circ \varphi})$ on $\operatorname{ch}(B) \backslash K$. In [7] the authors considered the compact case and (using a different method to [11]) showed that if $A$ is a uniform algebra on $X$ (or a certain closed subspace of $C(X)$ ) a real-linear isometry $T: A \rightarrow C(Y)$, whose range need not be a complex subspace of $C(Y)$, has the following description

$$
T f(y)=T 1(y)(\operatorname{Re}(f \circ \Phi)(y)+\alpha(y) i \operatorname{Im}(f \circ \Phi)(y)))
$$

for all $f \in A$ and $y \in Y_{0}$. Here $\Phi: Y_{0} \rightarrow \operatorname{ch}(A)$ and $\alpha: Y_{0} \rightarrow[-1,1]$ are continuous functions, where $\Phi$ is surjective. In particular, if $|T i|=1$ on $Y_{0}$ (this happens, for example, when $T(A)$ is a complex subspace of $C(Y))$ then $Y_{0}$ is a boundary for $T(A)$ and $\alpha$ takes its values in $\{-1,1\}$.

In [8] surjective real-linear isometries between complex subspaces of continuous functions on locally compact Hausdorff spaces have been studied. It is proven that, under some additional assumptions on separating conditions, such isometries are weighted composition 
operators on Choquet boundaries.

In this paper, we consider locally compact Hausdorff spaces $X$ and $Y$ and a real-linear isometry $T: A \rightarrow C_{0}(Y)$ for a complex subspace $A$ of $C_{0}(X)$ (without any additional assumption), and using the set of extremely strong boundary points of $A$ we introduce a subset $Y_{0}$ of $Y$ and give a description of $T$ on $Y_{0}$ (Theorem 3.5). We should note that here, the range of $T$ neither is a complex subspace nor satisfies any separating property. However, the results are improved in Theorems 3.6 and 3.10 under each of the following conditions;

i) $T(A)$ is a complex subspace of $C_{0}(Y)$ and $\Theta(A)=\operatorname{ch}(A)$ where $\Theta(A)$ and $\operatorname{ch}(A)$ are the set of extremely strong boundary points and the Choquet boundary of $A$, respectively or

ii) $T(A)$ satisfies a certain separating property.

\section{Preliminaries}

We recall that for a locally compact Hausdorff space $X, C_{0}(X)$ denotes the Banach space of all complex-valued continuous functions on $X$ vanishing at infinity, endowed with the supremum norm $\|\cdot\|_{\infty}$. For each point $x \in X, e_{x}$ is the evaluation functional at $x$.

We use briefly the notations $\mathbb{R}$-subspace, and $\mathbb{C}$-subspace for a real subspace and a complex subspace of $C_{0}(X)$, respectively. The complex dual of a $\mathbb{C}$-subspace $M$ of $C_{0}(X)$ (with respect to the supremum norm) will be denoted by $M_{\mathbb{C}}^{*}$ and, furthermore, $\Sigma_{\mathbb{C}}^{M}$ denotes the closed unit ball of $M_{\mathbb{C}}^{*}$. Similarly for an $\mathbb{R}$-subspace $M$ of $C_{0}(X), M_{\mathbb{R}}^{*}$ and $\Sigma_{\mathbb{R}}^{M}$ denote the real dual of $M$ and the closed unit ball of $M_{\mathbb{R}}^{*}$. We also use the notation ext(.) for the set of extreme points. It is well-known that for a $\mathbb{C}$-subspace $M$ of $C_{0}(X)$ every point in $\operatorname{ext}\left(\Sigma_{\mathbb{C}}^{M}\right)$ has the form $\alpha e_{x}$ for some $\alpha$ in the unit circle $\mathbb{T}$ and $x \in X$ (see for instance [4, Page 441]).

A boundary for an $\mathbb{R}$-subspace $M$ of $C_{0}(X)$ is a subset $E$ of $X$ having a non-empty intersection with the maximum set $M(f)=\left\{x \in X:|f(x)|=\|f\|_{\infty}\right\}$ of each $f \in M$. The peripheral range of a function $f \in C_{0}(X)$ denoted by $R_{\pi}(f)$ is the set $\{f(x): x \in M(f)\}$.

Let $M$ be a $\mathbb{C}$-subspace of $C_{0}(X)$. The Choquet boundary of $M$, denoted by $\operatorname{ch}(M)$, is defined as the set of all points $x \in X$ such that $e_{x} \in \operatorname{ext}\left(\Sigma_{\mathbb{C}}^{M}\right)$. It is well-known that $\operatorname{ch}(M)$ is a boundary for $M$ (see [15, Page 184]). A point $x \in X$ is called a strong boundary point of an $\mathbb{R}$-subspace $M$ of $C_{0}(X)$ if for each neighborhood $V$ of $x$ there exists a function $f \in M$ such that $|f(x)|=1=\|f\|_{\infty}$ and $|f|<1$ on $X \backslash V$. Evidently, in the case that $M$ is a $\mathbb{C}$-subspace, we may assume that the function $f$ in the above definition satisfies $f(x)=1$.

Following [3] a $\mathbb{C}$-subspace $M$ of $C_{0}(X)$ is called extremely regular if given $0<\varepsilon<1$, for each $x \in X$ and a neighborhood $V$ of $x$ there exists a function $f \in M$ with $f(x)=$ $\|f\|_{\infty}=1$ and $|f|<\varepsilon$ on $X \backslash V$ and extremely regular of type zero if for each $x \in X$ and a neighborhood $V$ of $x$ there exists a function $f \in M$ with $f(x)=\|f\|_{\infty}=1$ and $f=0$ on $X \backslash V$. Motivated by this, for an $\mathbb{R}$-subspace $M$ of $C_{0}(X)$ we call a point $x \in X$ an extremely strong boundary point of $M$ if for each neighborhood $V$ of $x$ and $0<\varepsilon \leq 1$ there exists a function $f \in M$ such that $\left|f\left(x_{0}\right)\right|=1=\|f\|_{\infty}$ and $|f|<\varepsilon$ on $X \backslash V$. So that strong boundary points of $M$ are the points $x \in X$ satisfying the above property for $\varepsilon=1$. 
For an $\mathbb{R}$-subspace $M$ of $C_{0}(X)$ we denote the set of all extremely strong boundary points of $M$ by $\Theta(M)$ and the set of all its strong boundary points (possibly an empty set) by $\tau(M)$. Clearly $\Theta(M) \subseteq \tau(M)$ and, moreover, $\tau(M)=\Theta(M)$ whenever $M$ is a subalgebra of $C_{0}(X)$. A uniform algebra on a locally compact Hausdorff space $X$ is a closed subalgebra $A$ of $C_{0}(X)$ which separates the points of $X$ and vanishing nowhere on $X$, in the sense that for each $x \in X$ there exists $f \in A$ with $f(x) \neq 0$. It is well-known that for a uniform algebra $A$ on a locally compact Hausdorff space $X, \operatorname{ch}(A)=\tau(A)$ (see [10, Theorem 4.7.22] for compact case and [14, Theorem 2.1] for locally compact case).

Clearly for a (unital) uniform algebra $A$ on a compact Hausdorff space $X$ and a strictly positive function $f_{0} \in C(X), A f_{0}$ is a closed subspace of $C(X)$. By Lemma 3.2 in [2] for each $x_{0} \in \operatorname{ch}(A)$ and strictly positive function $p \in C(X)$ there exists $f \in A$ with $f f_{0}\left(x_{0}\right)=p\left(x_{0}\right)$ and $\left|f f_{0}\right|<p$. This easily implies that every point in $\operatorname{ch}(A)$ is an extremely strong boundary point of $A f_{0}$, that is $\tau(A)=\operatorname{ch}(A) \subseteq \Theta\left(A f_{0}\right)$. In particular, if $A$ is regular, in the sense that $\tau(A)=X$, then $A f_{0}$ is extremely regular.

\section{Main Results}

We begin this section with two lemmas, which will be used in Theorem 3.5.

LEMMA 3.1. Let $X$ be a locally compact Hausdorff space and $A$ be a $\mathbb{C}$-subspace of $C_{0}(X)$. Then $\Theta(A) \subseteq \operatorname{ch}(A)$.

Proof. Let $x \in \Theta(A)$ and assume that $e_{x}=\frac{l_{1}+l_{2}}{2}$ for some $l_{1}, l_{2} \in \Sigma_{\mathbb{C}}^{A}$. Given $\varepsilon>0$, for each neighborhood $U$ of $x$ there exists, by hypothesis, a function $f \in A$ such that $f(x)=1=\|f\|_{\infty}$ and $|f|<\varepsilon$ on $X \backslash U$. Extending $l_{1}, l_{2}$ to continuous functionals on $C_{0}(X)$ with the same norm, we can find complex regular Borel measures $\mu_{1}, \mu_{2}$ on $X$ satisfying

$$
l_{i}(g)=\int_{X} g d \mu_{i} \quad(g \in A)
$$

for $i=1$, 2. Hence since $\|f\|_{\infty}=1$ it follows that

$$
1=f(x)=\frac{\int_{X} f d \mu_{1}+\int_{X} f d \mu_{2}}{2}=\left|\frac{\int_{X} f d \mu_{1}+\int_{X} f d \mu_{2}}{2}\right| \leq \frac{\left\|\mu_{1}\right\|+\left\|\mu_{2}\right\|}{2}
$$

that is $\left\|\mu_{1}\right\|=\left\|\mu_{2}\right\|=1$. Set $\mu=\frac{\mu_{1}+\mu_{2}}{2}$ then $\mu$ is a complex measure representing $e_{x}$ on $A$, i.e. $g(x)=\int_{X} g d \mu$ for all $g \in A$. Therefore,

$$
\begin{aligned}
1=\left|\int_{X} f d \mu\right| & \leq \int_{U}|f| d|\mu|+\int_{X \backslash U}|f| d|\mu| \\
& \leq|\mu|(U)+\varepsilon|\mu|(X \backslash U)
\end{aligned}
$$

and so $1 \leq|\mu|(U)+\varepsilon|\mu|(X \backslash U)$ for all $0<\varepsilon<1$ which implies that $|\mu|(U)=1$. Since $U$ is an arbitrary neighborhood of $x$ we conclude that $|\mu|$ is the point mass at $x$ and consequently 
$\mu$ is a modulus one multiple of the point mass. Now since $\mu=\frac{\mu_{1}+\mu_{2}}{2}$ and since the point mass at $x$ is an extreme point of the unit ball of $C_{0}(X)^{*}$ we get $\mu_{1}=\mu_{2}$. Therefore $l_{1}=l_{2}$, as desired.

For a $\mathbb{C}$-subspace $A$ of $C_{0}(X)$ and $x \in X$ we set $V_{x}=\left\{f \in A: f(x)=1=\|f\|_{\infty}\right\}$ which is clearly a nonempty set for each $x \in \tau(A)$. Next lemma states a similar additive version of Bishop's lemma given in [11, Lemma 3.14] and [16, Lemma 1] for the extremely strong boundary points of $\mathbb{C}$-subspaces of $C_{0}(X)$. The proof is basically the same (with a minor modification) and for the sake of completeness we state it here.

LemmA 3.2. Let $A$ be a closed $\mathbb{C}$-subspace of $C_{0}(X), x_{0} \in \Theta(A)$ and $f \in A$ with $\|f\|_{\infty}=1$ and $f\left(x_{0}\right)=0$. Then for each $0<\varepsilon<1$ there exists $u \in V_{x_{0}}$ such that $f+\frac{1}{1-\varepsilon} u \in \frac{1}{1-\varepsilon} V_{x_{0}}$.

ProOF. As in [11] we consider the following closed subsets of $X$

$$
\begin{aligned}
& F_{0}=\left\{x \in X:|f(x)| \geq \frac{1}{2}\right\}, \\
& F_{n}=\left\{x \in X: \frac{1}{2^{n+1}} \leq|f(x)| \leq \frac{1}{2^{n}}\right\} \quad(n \in \mathbb{N}) .
\end{aligned}
$$

Since $x_{0} \notin F_{n}, n \geq 0$, and $x_{0}$ is an extremely strong boundary point, it follows that for $0<\varepsilon<1$ and $n \geq 1$ we can find $u_{n} \in A$, such that $u_{n}\left(x_{0}\right)=1=\left\|u_{n}\right\|_{\infty}$ and $\left|u_{n}\right|<\varepsilon$ on $F_{0} \cup F_{n}$. Setting $u=\Sigma_{n=1}^{\infty} \frac{u_{n}}{2^{n}}$ we get a function $u \in A$ satisfying $u\left(x_{0}\right)=1=\|u\|_{\infty}$, that is $u \in V_{x_{0}}$. We now show that $f+\frac{1}{1-\varepsilon} u \in \frac{1}{1-\varepsilon} V_{x_{0}}$. For each $x \in F_{0}$ clearly we have

$$
\left|f(x)+\frac{1}{1-\varepsilon} u(x)\right| \leq|f(x)|+\frac{\varepsilon}{1-\varepsilon} \leq 1+\frac{\varepsilon}{1-\varepsilon}=\frac{1}{1-\varepsilon} .
$$

Assume now that $x \in F_{n_{0}}$ for some $n_{0} \geq 1$, then $|f(x)| \leq \frac{1}{2^{n_{0}}}$ and

$$
|u(x)| \leq \frac{\left|u_{n_{0}}(x)\right|}{2^{n_{0}}}+\Sigma_{n \neq n_{0}} \frac{\left|u_{n}(x)\right|}{2^{n}} \leq \frac{\varepsilon}{2^{n_{0}}}+1-\frac{1}{2^{n_{0}}}=1+\frac{\varepsilon-1}{2^{n_{0}}}
$$

and therefore

$$
\left|f(x)+\frac{1}{1-\varepsilon} u(x)\right| \leq \frac{1}{1-\varepsilon} .
$$

Finally for each $x \in X \backslash \cup_{n=0}^{\infty} F_{n}$, we have $f(x)=0$ and consequently $\left|f(x)+\frac{1}{1-\varepsilon} u(x)\right| \leq$ $\frac{1}{1-\varepsilon}$. This argument shows that $\left|f(x)+\frac{1}{1-\varepsilon} u(x)\right| \leq \frac{1}{1-\varepsilon}$ and since $\left|f\left(x_{0}\right)+\frac{1}{1-\varepsilon} u\left(x_{0}\right)\right|=\frac{1}{1-\varepsilon}$ it follows that $u$ has the desired properties.

In the sequel we assume that $X, Y$ are locally compact Hausdorff spaces, $A$ is a $\mathbb{C}$ subspace of $C_{0}(X)$ with $\Theta(A) \neq \emptyset$ and $T: A \rightarrow C_{0}(Y)$ is a real-linear isometry whose range need not be a $\mathbb{C}$-subspace of $C_{0}(Y)$. We set $B=T(A)$, which is clearly an $\mathbb{R}$-subspace 
of $C_{0}(Y)$. Since for each $\mathbb{C}$-subspace $M$ of $C_{0}(Y), l \rightarrow \operatorname{Re}(l)$ is a surjective isometry from $M_{\mathbb{C}}^{*}$ onto $M_{\mathbb{R}}^{*}$, it follows that $\operatorname{ext}\left(\Sigma_{\mathbb{R}}^{M}\right) \subseteq\left\{\operatorname{Re}\left(\alpha e_{x}\right): x \in X, \alpha \in \mathbb{T}\right\}$. Hence since the adjoint $T^{*}: B_{\mathbb{R}}^{*} \rightarrow A_{\mathbb{R}}^{*}$ of $T: A \rightarrow B$ is a surjective real-linear isometry, it follows that for each $x \in \operatorname{ch}(A)$ there exist $\beta \in \mathbb{T}$ and $y \in Y$ such that $T^{*}\left(\operatorname{Re}\left(\bar{\beta} e_{y}\right)\right)=\operatorname{Re}\left(e_{x}\right)$ which concludes that $\operatorname{Re}(\bar{\beta} T f(y))=\operatorname{Re}(f(x))$ for all $f \in A$. Now for each $x \in \operatorname{ch}(A)$ we set

$$
H_{x}=\left\{y \in Y: T^{*}\left(\operatorname{Re}\left(\bar{\beta} e_{y}\right)\right)=\operatorname{Re}\left(e_{x}\right) \text { for some } \beta \in \mathbb{T}\right\}
$$

which is a nonempty subset of $Y$.

LEMMA 3.3. For distinct points $x, x^{\prime} \in \Theta(A)$, we have $H_{x} \cap H_{x^{\prime}}=\emptyset$

PROOF. Let $x, x^{\prime}$ be distinct points of $\Theta(A)$ and assume on the contrary that there exists $y \in H_{x} \cap H_{x^{\prime}}$. Then there are scalars $\beta, \gamma \in \mathbb{T}$ such that

$$
T^{*}\left(\operatorname{Re}\left(\bar{\beta} e_{y}\right)\right)=\operatorname{Re}\left(e_{x}\right), \quad T^{*}\left(\operatorname{Re}\left(\bar{\gamma} e_{y}\right)\right)=\operatorname{Re}\left(e_{x^{\prime}}\right) .
$$

Hence for each $f \in A$,

$$
\operatorname{Re}(\bar{\beta} T f(y))=\operatorname{Re}(f(x)), \quad \operatorname{Re}(\bar{\gamma} T f(y))=\operatorname{Re}\left(f\left(x^{\prime}\right)\right) .
$$

Since $x \in \Theta(A)$ and $x \neq x^{\prime}$, for each $0<\varepsilon<1$ we can find a function $f_{0} \in A$ such that $f_{0}(x)=1=\left\|f_{0}\right\|_{\infty}$ and $\left|f_{0}\left(x^{\prime}\right)\right|<\varepsilon$. Then clearly $T f_{0}(y)=\beta$, since $\left\|T f_{0}\right\|_{\infty}=\left\|f_{0}\right\|_{\infty}=$ 1 , and $\left|\operatorname{Re}\left(\bar{\gamma} T f_{0}(y)\right)\right|<\varepsilon$ that is $\operatorname{Re}(\bar{\gamma} \beta)<\varepsilon$. Being $\varepsilon>0$ arbitrary we get $\operatorname{Re}(\bar{\gamma} \beta)=0$, i.e. $\beta= \pm i \gamma$. Assume without loss of generality that $\beta=i \gamma$, then

$$
\operatorname{Re}(\bar{\beta} T f(y))=\operatorname{Re}(f(x)), \quad \operatorname{Re}(i \bar{\beta} T f(y))=\operatorname{Re}\left(f\left(x^{\prime}\right)\right)
$$

for all $f \in A$. Let $0<\varepsilon<1$ be given and $f_{0} \in A$ be as above, i.e. $f_{0}(x)=1=\left\|f_{0}\right\|_{\infty}$ and $\left|f_{0}\left(x^{\prime}\right)\right|<\varepsilon$ and let $F=\left\{z \in X:\left|f_{0}(z)\right| \geq \varepsilon\right\}$. Then $F$ is a nonempty closed subset of $X$ and since $x^{\prime} \notin F$ we can find $h \in A$ with $h\left(x^{\prime}\right)=1=\|h\|_{\infty}$ and $|h|<\varepsilon$ on $F$. It is now easy to see that $\left\|f_{0}+h\right\|_{\infty}<1+\varepsilon$ and since $T f_{0}(y)=\beta, T h(y)=-i \beta$ it follows that

$|1-i|=|\beta-i \beta|=\left|T f_{0}(y)+T h(y)\right|=\left|T\left(f_{0}+h\right)(y)\right| \leq\left\|T\left(f_{0}+h\right)\right\|_{\infty}=\left\|f_{0}+h\right\|_{\infty}<1+\varepsilon$,

which is impossible, since $\varepsilon$ is arbitrary.

Setting $Y_{0}=\bigcup_{x \in \Theta(A)} H_{x}$, the above lemma allows us to define a map $\Phi: Y_{0} \rightarrow \Theta(A)$ such that for each $y \in Y_{0}, \Phi(y)$ is the unique point $x \in \Theta(A)$ with $y \in H_{x}$. Clearly $\Phi$ is a well-defined surjective map.

LEMMA 3.4. i) For each $y \in Y_{0}$ and $f \in A, f(\Phi(y))=0$ implies $T f(y)=0$.

ii) There exists a function $\alpha: Y_{0} \rightarrow[-1,1]$ such that

$$
T(i f)(y)=\alpha(y) i T f(y), \quad\left(y \in Y_{0}, f \in V_{\Phi(y)}\right) .
$$

Proof. i) Let $y \in Y_{0}$ and $f \in A$ with $f(\Phi(y))=0$. Assume without loss of generality that $\|f\|_{\infty}=1$. For $\varepsilon=\frac{1}{2}$ and $x=\Phi(y)$ let $u \in V_{x}$ be chosen as in Lemma 
3.2. Then since $T\left(V_{x}\right) \subseteq\left\{g \in C_{0}(Y):\|g\|_{\infty}=1, g(y)=\beta\right\}$ for some $\beta \in \mathbb{T}$, we have $T u(y)=\beta$ and $T(f+2 u)(y)=2 \beta$ which implies that $T f(y)=0$.

ii) Let $y \in Y_{0}$. Then there exists $\beta \in \mathbb{T}$ such that $\operatorname{Re}(\bar{\beta} T f(y))=\operatorname{Re}(f(x))$ for all $f \in$ $A$, where $x=\Phi(y)$. Fixing a function $f_{0} \in V_{x}$ we have $T f_{0}(y)=\beta$ and $\operatorname{Re}\left(\bar{\beta} T\left(i f_{0}\right)\right)(y)=0$ and consequently $T\left(i f_{0}\right)(y)=i \alpha(y) \beta=i \alpha(y) T f_{0}(y)$ for some $\alpha(y) \in[-1,1]$. The scalar $\alpha(y)$ is independent of the function $f_{0}$ in $V_{x}$, since for each $f \in V_{x},\left(f-f_{0}\right)(x)=0=$ $i\left(f-f_{0}\right)(x)$ and so, by (i), $T\left(f-f_{0}\right)(y)=0=T\left(i f-i f_{0}\right)(y)$, that is $T f(y)=T f_{0}(y)$ and $T(i f)(y)=T\left(i f_{0}\right)(y)$, as desired.

THEOREM 3.5. Let $X, Y$ be locally compact Hausdorff spaces, $A$ be a $\mathbb{C}$-subspace of $C_{0}(X)$ with $\Theta(A) \neq \emptyset$. Then for a real-linear isometry $T: A \rightarrow C_{0}(Y)$ there exists a subset $Y_{0}$ of $Y$ and continuous functions $\Phi: Y_{0} \rightarrow \Theta(\bar{A}), \alpha: Y_{0} \rightarrow[-1,1]$ and $w: Y_{0} \rightarrow \mathbb{T}$, where $\Phi$ is surjective, such that

$$
T f(y)=w(y) \cdot(\operatorname{Re}(f(\Phi(y)))+\alpha(y) i \operatorname{Im}(f(\Phi(y)))
$$

for all $f \in A$ and $y \in Y_{0}$.

Proof. Since $T$ can be extended to a real-linear isometry from the closure $\bar{A}$ of $A$ into $C_{0}(Y)$ we may assume without loss of generality that $A$ is closed. Let $B=T(A)$ and let the subset $Y_{0}$ of $Y$ and the functions $\alpha: Y_{0} \rightarrow[-1,1]$ and $\Phi: Y_{0} \rightarrow \Theta(A)$ be defined as above. By definition, $\Phi$ is surjective. For a given $y \in Y_{0}$ let $x=\Phi(y)$ and set $w(y)=T h(y)$ where $h \in V_{x}$ is an arbitrary function. As before $w(y)$ does not depend on the function $h$ in $V_{x}$ and, moreover, it follows from the definition of $\alpha$ that $T(i h)(y)=\alpha(y) i T h(y)$. Clearly (1) holds for all $f \in A$ with $f(x)=0$. Hence for each $f \in A$, since the function $g=f-f(x) h$ satisfies $g(x)=0$, it follows from Lemma 3.4(i) that $T g(y)=0$ and so by the real-linearity of $T$

$$
\begin{aligned}
T f(y)=T(f(x) h)(y) & =T(\operatorname{Re}(f(x)) h)(y)+T(\operatorname{Im}(f(x)) i h)(y) \\
& =\operatorname{Re}(f(x)) T h(y)+\operatorname{Im}(f(x)) T(i h)(y) \\
& =\operatorname{Re}(f(x)) T h(y)+\operatorname{Im}(f(x)) \alpha(y) i T(h)(y) \\
& =w(y) \cdot(\operatorname{Re}(f(x))+\alpha(y) i \operatorname{Im}(f(x))) .
\end{aligned}
$$

To prove the continuity of $\Phi$, let $y \in Y_{0}$ and $U$ be a neighborhood of of $\Phi\left(y_{0}\right)$ in $\Theta(A)$. Let $\widetilde{U}$ be a neighborhood in $X$ with $U=\widetilde{U} \cap \Theta(A)$ and for arbitrary $0<r<1$ let $f \in V_{\Phi\left(y_{0}\right)}$ such that $|f| \leq r$ on $X \backslash \widetilde{U}$. Then $O=\left\{y \in Y_{0}:|T f(y)|>r\right\}$ is a neighborhood of $y_{0}$ in $Y_{0}$, since $f \in V_{\Phi\left(y_{0}\right)}$ and $T\left(V_{\Phi\left(y_{0}\right)}\right) \subseteq \beta\left\{g \in C_{0}(Y): g\left(y_{0}\right)=1=\|g\|_{\infty}\right\}$ for some $\beta \in \mathbb{T}$. Moreover, for each $y \in O$

$$
|f(\Phi(y))| \geq|\operatorname{Re}(f(\Phi(y)))+\alpha(y) i \operatorname{Im}(f(\Phi(y)))|=|T f(y)|>r
$$

that is, $\Phi(O) \subseteq \widetilde{U} \cap \Theta(A)$ and so $\Phi$ is continuous.

We now show that $w$ is continuous. For this, suppose that $\left\{y_{\lambda}\right\}$ is a net in $Y_{0}$ converging to $y_{0} \in Y_{0}$ and choose arbitrary functions $h_{0} \in V_{\Phi\left(y_{0}\right)}$ and $h_{\lambda} \in V_{\Phi\left(y_{\lambda}\right)}$ for all $\lambda$. Then 
since $\left(h_{\lambda}-h_{0}\right)\left(\Phi\left(y_{\lambda}\right)\right)=1-h_{0}\left(\Phi\left(y_{\lambda}\right)\right) \rightarrow 0$, the given description of $T$ shows that $T\left(h_{\lambda}-h_{0}\right)\left(y_{\lambda}\right) \rightarrow 0$ which easily implies that $w\left(y_{\lambda}\right) \rightarrow w\left(y_{0}\right)$, that is $w$ is continuous.

Now it suffices to show that $\alpha: Y_{0} \rightarrow[-1,1]$ is continuous. For this let $y_{0} \in Y_{0}$ and let $f$ be an arbitrary function in $V_{\Phi\left(y_{0}\right)}$. Then since if $\left(\Phi\left(y_{0}\right)\right)=i$, there exists a neighborhood $U$ of $y_{0}$ in $Y_{0}$ such that $\operatorname{Im}(i f(\Phi(y))) \neq 0$ for all $y \in U$. Now since for each $z \in Y_{0}$, $T(i f)(z)=w(z) \cdot(\operatorname{Re}(i f(\Phi(z)))+\alpha(z) i \operatorname{Im}(i f(\Phi(z)))$ it follows that for each $y \in U$

$$
\alpha(y)=\frac{T(i f)(y)-w(y) \cdot \operatorname{Re}(i f(\Phi(y)))}{w(y) i \operatorname{Im}(i f(\Phi(y)))}
$$

which implies that $\alpha$ is continuous.

In the next theorem we improve the above description of $T$ under the additional assumptions that $T(A)$ is a $\mathbb{C}$-subspace and $\Theta(A)=\operatorname{ch}(A)$. This extends [7, Theorem 3.4] and [11, Theorem 1.1]. On the other hand, since by [8, Proposition 4.1] each $\mathbb{C}$-subspace $A$ of $C_{0}(X)$ with $\Theta(A)=\operatorname{ch}(\mathrm{A})$ is strongly separating and strongly 0 -separating in the sense which are defined in [8], Theorem 1.1 in [8], in particular, gives a description of a surjective real-linear isometry $T: A \rightarrow B$ between $\mathbb{C}$-subspaces $A$ and $B$ such that $\Theta(A)=\operatorname{ch}(A)$ and $B$ is strongly separating. Hence the following theorem may also be considered as a generalization of this particular case of [8, Theorem 1.1] by removing the separating assumption on $T(A)$.

THEOREM 3.6. Let $X, Y$ be locally compact Hausdorff spaces, $A$ be a $\mathbb{C}$-subspace of $C_{0}(X)$ such that $\Theta(A)=\operatorname{ch}(\mathrm{A})$. If $T: A \rightarrow C_{0}(Y)$ is a real-linear isometry whose range is a $\mathbb{C}$-subspace of $C_{0}(Y)$, then there are a subset $Y_{0}$ of $Y$ which is a boundary for $T(A)$, continuous functions $\Phi: Y_{0} \rightarrow \operatorname{ch}(A), w: Y_{0} \rightarrow \mathbb{T}$, where $\Phi$ is surjective, and a clopen subset $K$ of $Y_{0}$ such that

$$
T f(y)=w(y) \begin{cases}\frac{f(\Phi(y))}{f(\Phi(y))} & y \in K, \\ y \in Y_{0} \backslash K,\end{cases}
$$

for each $f \in A$ and $y \in Y_{0}$.

Proof. We first note that since $\operatorname{ch}(\bar{A}) \supseteq \Theta(\bar{A}) \supseteq \Theta(A)$ and clearly $\operatorname{ch}(A)=\operatorname{ch}(\bar{A})$, it follows from the hypotheses that $\Theta(\bar{A})=\operatorname{ch}(\bar{A})$. Hence as before, by extending $T$ on $\bar{A}$, we can assume that $A$ is closed in $C_{0}(X)$. Let $B=T(A)$ and let $Y_{0}, \Phi, w$ and $\alpha$ be defined as in Theorem 3.5. Then

$$
T f(y)=w(y) \cdot(\operatorname{Re}(f(\Phi(y)))+\alpha(y) i \operatorname{Im}(f(\Phi(y)))
$$

for all $f \in A$ and $y \in Y_{0}$. Since $B$ is assumed to be a $\mathbb{C}$-subspace of $C_{0}(Y)$, the definition of $Y_{0}$ shows that for each $y \in Y_{0}, e_{y} \in \operatorname{ext}\left(\Sigma_{\mathbb{C}}^{B}\right)$ and hence $Y_{0} \subseteq \operatorname{ch}(B)$. We shall show that $\alpha(y) \in\{-1,1\}$ for all $y \in Y_{0}$. Let $y \in Y_{0}$. Then there exist $\beta \in \mathbb{T}$ and $x \in \Theta(A)$ such that $\operatorname{Re}(\bar{\beta} T f(y))=\operatorname{Re}(f(x))$ for all $f \in A$. Let $\lambda=i \bar{\beta}$. Then since $\lambda e_{y} \in \operatorname{ext}\left(\Sigma_{\mathbb{C}}^{B}\right)$ and $\operatorname{ch}(\mathrm{A})=\Theta(A)$, there exists a point $x^{\prime} \in \Theta(A)$ and $\gamma \in \mathbb{T}$ such that

$$
\operatorname{Re}(\lambda T f(y))=\operatorname{Re}\left(\gamma f\left(x^{\prime}\right)\right)
$$


for all $f \in A$. We now show that $x=x^{\prime}$. Since $\frac{\bar{\beta}+\lambda}{\sqrt{2}} e_{y} \in \operatorname{ext}\left(\Sigma_{\mathbb{C}}^{B}\right)$, there exist an $x^{\prime \prime} \in \Theta(A)$ and a $\mu \in \mathbb{T}$ such that $\operatorname{Re}((\bar{\beta}+\lambda) T f(y))=\sqrt{2} \operatorname{Re}\left(\mu f\left(x^{\prime \prime}\right)\right)$ for all $f \in A$. Hence

$$
\operatorname{Re}(f(x))+\operatorname{Re}\left(\gamma f\left(x^{\prime}\right)\right)=\sqrt{2} \operatorname{Re}\left(\mu f\left(x^{\prime \prime}\right)\right)
$$

for all $f \in A$. If $x, x^{\prime}$ and $x^{\prime \prime}$ are distinct, then there exists an $f_{0} \in V_{x}$ such that $\left|f_{0}\left(x^{\prime}\right)\right|<1 / 4$ and $\sqrt{2}\left|f_{0}\left(x^{\prime \prime}\right)\right|<1 / 4$. Therefore,

$$
1=\operatorname{Re}\left(f_{0}(x)\right)=\sqrt{2} \operatorname{Re}\left(\mu f_{0}\left(x^{\prime \prime}\right)\right)-\operatorname{Re}\left(\gamma f_{0}\left(x^{\prime}\right)\right) \leq \sqrt{2}\left|f_{0}\left(x^{\prime \prime}\right)\right|+\left|f_{0}\left(x^{\prime}\right)\right|<\frac{1}{2},
$$

which is impossible. Consequently, one of the equalities $x=x^{\prime}, x=x^{\prime \prime}$ and $x^{\prime}=x^{\prime \prime}$ is true. To prove $x=x^{\prime}$, we consider only the case where $x=x^{\prime \prime}$. In this case $\operatorname{Re}\left(\gamma f\left(x^{\prime}\right)\right)=$ $\operatorname{Re}((\sqrt{2} \mu-1) f(x))$ for all $f \in A$. If $x \neq x^{\prime}$, then $\left|(\sqrt{2} \mu-1) f_{0}(x)\right|<1$ for some $f_{0} \in V_{x^{\prime}}$. Thus

$$
1=f_{0}\left(x^{\prime}\right)=\operatorname{Re}\left((\sqrt{2} \mu-1) \bar{\gamma} f_{0}(x)\right) \leq\left|(\sqrt{2} \mu-1) f_{0}(x)\right|<1,
$$

a contradiction. This shows that $x=x^{\prime}$. By the same reasoning, we see that if $x^{\prime}=x^{\prime \prime}$, then $x=x^{\prime}$.

Now, choosing $f \in A$ with $f(x)=1=\|f\|_{\infty}$ (that is, $f \in V_{x}$ ) since $x=x^{\prime}$ we have

$$
\operatorname{Re}(\lambda T f(y))=\operatorname{Re}(\gamma), \quad \operatorname{Re}(\lambda T(i f)(y))=-\operatorname{Im}(\gamma) .
$$

Therefore by the definition of $\alpha$

$$
\lambda T(i f)(y)=\alpha(y) i \lambda T f(y)=\alpha(y) i(\operatorname{Re}(\gamma)+i \operatorname{Im}(\lambda T f(y)))
$$

and so $\operatorname{Im}(\gamma)=-\operatorname{Re}(\lambda T(i f)(y))=\alpha(y) \operatorname{Im}(\lambda T f(y))$. Thus

$$
\begin{aligned}
\operatorname{Re}(\lambda T f(y))^{2}+\alpha(y)^{2} \operatorname{Im}(\lambda T f(y))^{2} & =\operatorname{Re}(\gamma)^{2}+\operatorname{Im}(\gamma)^{2}=1 \\
& =|\lambda T f(y)|^{2}=\operatorname{Re}(\lambda T f(y))^{2}+\operatorname{Im}(\lambda T f(y))^{2} .
\end{aligned}
$$

Therefore $\alpha(y)^{2}=1$, since $\operatorname{Im}(\lambda T f(y))=\operatorname{Im}(\lambda \beta) \neq 0$, that is $\alpha(y) \in\{-1,1\}$.

The above argument shows that the set $K=\left\{y \in Y_{0}: \alpha(y)=1\right\}$ is a clopen subset of $Y_{0}$ and, moreover, for each $y \in Y_{0}$ and $f \in A$

$$
(T f)(y)=(T 1)(y) \begin{cases}\frac{f(\Phi(y))}{f(\Phi(y))} & y \in K, \\ y \in Y_{0} \backslash K .\end{cases}
$$

Now since $\operatorname{ch}(A)$ is a boundary for $A$ and $\phi: Y_{0} \rightarrow \operatorname{ch}(A)$ is surjective it follows that $Y_{0}$ is a boundary for $B$, as desired.

COROLlary 3.7. Let $X, Y$ be compact Hausdorff spaces, $A$ be a uniform algebra on $X$ and $B$ be $a \mathbb{C}$-subspace of $C(Y)$. If $T: A \rightarrow B$ is a surjective map satisfying $R_{\pi}(T f+$ $T g)=R_{\pi}(f+g)$ for all $f, g \in A$, then there are a subset $Y_{0}$ of $Y$, which is a boundary for $B$, and a continuous surjective map $\Phi: Y_{0} \rightarrow \operatorname{ch}(A)$ such that $T f(y)=f(\Phi(y))$ for all $f \in A$ and $y \in Y_{0}$. In particular, $T$ is complex-linear. 
Proof. Using the Mazur-Ulam Theorem, the hypotheses imply that $T$ is a real-linear isometry from $A$ onto $B$. We note that since $A$ is a uniform algebra on $X, \Theta(A)=\operatorname{ch}(A)$. Let $Y_{0}, K$ and $\Phi: Y_{0} \rightarrow \operatorname{ch}(A)$ be as in the above theorem. Then since $R_{\pi}(T 1)=R_{\pi}(1)=$ $\{1\}$ and $|T 1|=1$ on $Y_{0}$ we conclude that $T 1=1$ on $Y_{0}$. The description of $T$ given in this theorem together with the fact that $R_{\pi}(T i)=R_{\pi}(i)=\{i\}$ shows that $K=Y_{0}$ and consequently $T f(y)=f(\Phi(y))$ for all $f \in A$ and $y \in Y_{0}$. It is now easy to see that $T$ is complex-linear, since $Y_{0}$ is a boundary for $B$.

In the next theorem we give a description of a real-linear isometry $T: A \rightarrow C_{0}(Y)$ whenever the range of $T$ satisfies a certain separating property which we call it $\mathbb{T}$-separating property.

Definition 3.8. Let $Y$ be a locally compact Hausdorff space. We say that an $\mathbb{R}$ subspaces $B$ of $C_{0}(Y)$ is $\mathbb{T}$-separating if for distinct points $y, y^{\prime} \in Y$ and scalars $\lambda, \lambda^{\prime} \in \mathbb{T}$ there exists a function $g \in B$ such that $\|g\|_{\infty}=1, g(y)=\lambda, g\left(y^{\prime}\right)=\lambda^{\prime}$.

It is easy to see that every extremely regular $\mathbb{C}$-subspace of $C_{0}(Y)$ of type zero is $\mathbb{T}$ separating and hence every $\mathbb{R}$-subspace of $C_{0}(Y)$ containing such subspace is $\mathbb{T}$-separating, as well. By [3] if $\mu$ is a non-zero regular Borel measure on a locally compact Hausdorff space $Y$ which is continuous (i.e. the atomic part of $\mu$ is zero), then the kernel $K(\mu)$ of $\mu$ is a (maximal) extremely regular subspace of type zero. Thus for each $f_{0} \in C_{0}(Y) \backslash K(\mu)$, the real subspace generated by $K(\mu)$ and $f_{0}$ is a $\mathbb{T}$-separating $\mathbb{R}$-subspace of $C_{0}(Y)$.

We should note that if $B$ is a $\mathbb{T}$-separating subspace of $C_{0}(Y)$, then given distinct points $y, y^{\prime} \in Y$ and scalar $\beta \in \mathbb{T}$ we can find $g \in B$ such that $\|g\|_{\infty}=1, g(y)=\beta$ and $g\left(y^{\prime}\right)=0$. In fact, it suffices to consider $g=\frac{g_{1}+g_{2}}{2}$ where $g_{1}, g_{2} \in B$ are norm one elements with $g_{1}(y)=g_{2}(y)=\beta, g_{1}\left(y^{\prime}\right)=\gamma$ and $g_{2}\left(y^{\prime}\right)=-\gamma$ for $\gamma \in \mathbb{T} \backslash\{ \pm \beta\}$.

Lemma 3.9. Let $X, Y$ be locally compact Hausdorff spaces, $A$ be a $\mathbb{C}$-subspace of $C_{0}(X), B$ be a $\mathbb{T}$-separating $\mathbb{R}$-subspace of $C_{0}(Y)$ and $T: A \rightarrow B$ be a surjective real-linear isometry. Then for each $x_{0} \in \tau(A) \cap \operatorname{ch}(A), H_{x_{0}}$ is a singleton and $H_{x_{0}}=\bigcap_{f \in V_{x_{0}}} M(T f)$.

Proof. Fix a point $x_{0} \in \tau(A) \cap \operatorname{ch}(A)$. We first show that $H_{x_{0}}$ is a singleton. For suppose that $y_{1}, y_{2} \in H_{x_{0}}$ be distinct and let $\beta, \gamma \in \mathbb{T}$ such that $T^{*}\left(\operatorname{Re}\left(\bar{\beta} e_{y_{1}}\right)\right)=\operatorname{Re}\left(e_{x_{0}}\right)$ and $T^{*}\left(\operatorname{Re}\left(\bar{\gamma} e_{y_{2}}\right)\right)=\operatorname{Re}\left(e_{x_{0}}\right)$. Then

$$
\operatorname{Re}\left(\bar{\beta} T f\left(y_{1}\right)\right)=\operatorname{Re}\left(f\left(x_{0}\right)\right), \quad \operatorname{Re}\left(\bar{\gamma} T f\left(y_{2}\right)\right)=\operatorname{Re}\left(f\left(x_{0}\right)\right)
$$

for all $f \in A$. Since $B$ is $\mathbb{T}$-separating there exists $f \in A$ such that $\|T f\|_{\infty}=1$ and $T f\left(y_{1}\right)=T f\left(y_{2}\right)=1$, so using (1) it follows that $\left.\operatorname{Re}(\bar{\gamma})\right)=\operatorname{Re}(\bar{\beta})$. Similarly there exists $f^{\prime} \in A$ such that $\left\|T f^{\prime}\right\|_{\infty}=1$ and $T f^{\prime}\left(y_{1}\right)=T f^{\prime}\left(y_{2}\right)=i$ which conclude that $\operatorname{Im}(\bar{\gamma})=$ $\operatorname{Im}(\bar{\beta})$. Therefore, $\gamma=\beta$ and consequently $\operatorname{Re}\left(\bar{\beta} T f\left(y_{1}\right)\right)=\operatorname{Re}\left(\bar{\beta} T f\left(y_{2}\right)\right)$ for all $f \in A$ which is impossible, since $B$ is $\mathbb{T}$-separating. Hence the set $H_{x_{0}}$ is a singleton.

We now show that $H_{x_{0}}=\bigcap_{f \in V_{x_{0}}} M(T f)$. Setting $Q_{x_{0}}=\bigcap_{f \in V_{x_{0}}} M(T f)$ we have clearly $H_{x_{0}} \subseteq Q_{x_{0}}$. Let $H_{x_{0}}=\left\{y_{0}\right\}$ and $y \in Q_{x_{0}} \backslash H_{x_{0}}$. Then there exists $\beta \in \mathbb{T}$ such that 
$T^{*}\left(\operatorname{Re}\left(\bar{\beta} e_{y_{0}}\right)\right)=\operatorname{Re}\left(e_{x_{0}}\right)$ and since $B$ is $\mathbb{T}$-separating we can find a function $f \in A$ such that $\|T f\|_{\infty}=1, T f\left(y_{0}\right)=\beta$ and $T f(y)=0$. Since $\|f\|_{\infty}=\|T f\|_{\infty}=1$ and $1=$ $\operatorname{Re}\left(\bar{\beta} T f\left(y_{0}\right)\right)=\operatorname{Re}\left(f\left(x_{0}\right)\right)$ it follows that $f\left(x_{0}\right)=1$ and hence $f \in V_{x_{0}}$ while $T f(y)=0$ which contradicts to $y \in Q_{x_{0}}$. Therefore, $H_{x_{0}}=Q_{x_{0}}$.

We should note that the notion of $\mathbb{T}$-separating, defined above, implies the condition which is considered in [8, Proposition 4.4]. By [8, Proposition 4.4 and Theorem 2.3] onto real-linear isometries $T: A \rightarrow B$ between $\mathbb{C}$-subspaces $A$ and $B$ are weighted composition operators provided that $A$ is strongly separating and satisfies this condition. However, in the next theorem we obtain a similar description for $T$ without any separating condition on $A$ whenever the range of $T$ (as an $\mathbb{R}$-subspace) is $\mathbb{T}$-separating.

THEOREM 3.10. Let $X, Y$ be locally compact Hausdorff spaces, $A$ be a $\mathbb{C}$-subspace of $C_{0}(X)$ with $\tau(A) \cap \operatorname{ch}(A) \neq \emptyset, B$ be a $\mathbb{T}$-separating $\mathbb{R}$-subspace of $C_{0}(Y)$ and $T: A \rightarrow B$ be a surjective real-linear isometry. Then there exist a subset $Y_{1}$ of $\tau(B)$, a clopen subset $K$ of $Y_{1}$ and continuous functions $\Phi: Y_{1} \rightarrow \tau(A) \cap \operatorname{ch}(A)$ and $w: Y_{1}: \rightarrow \mathbb{T}$, where $\Phi$ is bijective, such that

$$
(T f)(y)=w(y) \begin{cases}\frac{f(\Phi(y))}{f(\Phi(y))} & y \in K, \\ y \in Y_{1} \backslash K,\end{cases}
$$

for each $f \in A$ and $y \in Y_{1}$.

Proof. We set $Y_{1}=\bigcup_{x \in \tau(A) \cap \operatorname{ch}(A)} H_{x}$. For each $x \in \tau(A) \cap \operatorname{ch}(A), H_{x}$ is a singleton, by the above lemma. We first show that $Y_{1} \subseteq \tau(B)$. Let $y_{0} \in Y_{1}$ and $U$ be a neighborhood of $y_{0}$ in $Y$. Then $y_{0} \in H_{x_{0}}$ for some $x_{0} \in \tau(A) \cap \operatorname{ch}(A)$, that is $H_{x_{0}}=\left\{y_{0}\right\}$. Since $H_{x_{0}}=$ $\bigcap_{f \in V_{x_{0}}} M(T f)$ it follows that $\cap_{f \in V_{x_{0}}} M(T f) \cap\left(Y_{\infty} \backslash U\right)=\bigcap_{f \in V_{x_{0}}} M(T f) \cap(Y \backslash U)=\emptyset$ where $Y_{\infty}$ is the one-point compactification of $Y$. Therefore, there is $n \in \mathbb{N}$ and $f_{i} \in V_{x_{0}}$, $i=1, \ldots, n$ such that $\cap_{i=1}^{n} M\left(T f_{i}\right) \subseteq U$. Set $f=\Sigma_{i=1}^{n} \frac{f_{i}}{n}$, then clearly $f \in V_{x_{0}},\left|T f\left(y_{0}\right)\right|=$ $1=\|T f\|_{\infty}$ and since for each $i=1, \ldots, n,\left|T f_{i}\right|<1$ on $Y \backslash U$ we get $|T f|<1$ on $Y \backslash U$ and hence $y_{0}$ is a strong boundary point of $B$, that is $Y_{1} \subseteq \tau(B)$.

Using the $\mathbb{T}$-separating property of $B$, the same argument as in the proof of [7, Theorem 3.8] can be applied to show that if $x \in \tau(A) \cap \operatorname{ch}(A)$ and $f \in A$ such that $f(x)=0$, then $T f(y)=0$ where $y$ is the unique point of $H_{x}$. We now show that for distinct points $x, x^{\prime} \in \tau(A) \cap \operatorname{ch}(A), H_{x} \cap H_{x^{\prime}}=\emptyset$. Assume on the contrary that there exists a point $y \in H_{x} \cap H_{x^{\prime}}$, that is $H_{x}=H_{x^{\prime}}=\{y\}$. Then there exist scalars $\beta, \gamma \in \mathbb{T}$ such that

$$
\operatorname{Re}(\bar{\beta} T h(y))=\operatorname{Re}(h(x)) \text { and } \operatorname{Re}(\bar{\gamma} T h(y))=\operatorname{Re}\left(h\left(x^{\prime}\right)\right)
$$

holds for all $h \in A$. Since $x$ and $x^{\prime}$ are strong boundary points of $A$ and $A$ is a $\mathbb{C}$-subspace we can easily find an element $h \in A$ such that $h(x)=0$ and $h\left(x^{\prime}\right) \in \mathbb{R} \backslash\{0\}$. Therefore, by the above argument $T h(y)=0$ and this contradicts to (1). Hence, $H_{x} \cap H_{x^{\prime}}=\emptyset$. This allows us to define a map $\Phi: Y_{1} \rightarrow \tau(A) \cap \operatorname{ch}(A)$ which associates to each $y \in Y_{1}$ the unique point $x \in \tau(A) \cap \operatorname{ch}(A)$ with $H_{x}=\{y\}$. Clearly $\Phi$ is a bijective map. 
Now as in the proof of Lemma 3.4 and Theorem 3.5, we can define continuous functions $\alpha: Y_{1} \rightarrow[-1,1]$ and $w: Y_{1} \rightarrow \mathbb{T}$ such that $\alpha(y)=\frac{T(i f)(y)}{i T f(y)}$ for all $y \in Y_{1}$ and $f \in V_{\Phi(y)}$ and

$$
T f(y)=w(y) \cdot(\operatorname{Re}(f(\Phi(y)))+\alpha(y) i \operatorname{Im}(f(\Phi(y))))
$$

for all $f \in A$ and $y \in Y_{1}$. So it suffices to show that $\alpha(y) \in\{-1,1\}$ for all $y \in Y_{1}$. Given $y \in Y_{1}$, let $x=\Phi(y)$. Since $e_{x}, i e_{x} \in \operatorname{ext}\left(\Sigma_{\mathbb{C}}^{A}\right)$ we have $\operatorname{Re}\left(e_{x}\right), \operatorname{Re}\left(i e_{x}\right) \in \operatorname{ext}\left(\Sigma_{\mathbb{R}}^{A}\right)$. Therefore there exist $y \in Y_{1}, y^{\prime} \in Y$ and $\beta, \gamma \in \mathbb{T}$ such that

$$
T^{*}\left(\operatorname{Re}\left(\bar{\beta} e_{y}\right)\right)=\operatorname{Re}\left(e_{x}\right), \quad T^{*}\left(\operatorname{Re}\left(\bar{\gamma} e_{y^{\prime}}\right)\right)=R\left(i e_{x}\right) .
$$

Since $B$ is $\mathbb{T}$-separating one can show easily that $y=y^{\prime}$ and consequently

$$
\operatorname{Re}(\bar{\beta} T f(y))=\operatorname{Re}(f(x)), \quad \operatorname{Re}(\bar{\gamma} T f(y))=\operatorname{Re}(i f(x))
$$

for all $f \in A$. Now since $x$ is a strong boundary point of $A$ we can find $g \in A$ such that $g(x)=1=\|g\|_{\infty}$ and therefore $\operatorname{Re}(\bar{\beta} T g(y))=1$ and $\operatorname{Re}(\bar{\gamma} T(i g)(y))=-\operatorname{Re}(g(x))=-1$. Thus $T g(y)=\beta$ and $T(i g)(y)=-\gamma$. This implies that $\alpha(y)=\frac{-\gamma}{i \beta}$ and so $|\alpha(y)|=1$, i.e. $\alpha(y) \in\{-1,1\}$, as desired.

THEOREM 3.11. Let I be an arbitrary set, $X, Y$ be locally compact Hausdorff spaces, $A$ be $a \mathbb{C}$-subspace of $C_{0}(X)$ with $\Theta(A) \neq \emptyset$ and $B$ be an $\mathbb{R}$-subspace of $C_{0}(Y)$. Assume that $S_{1}, S_{2}: I \rightarrow A$ and $T_{1}, T_{2}: I \rightarrow B$ are surjective maps satisfying

$$
\left.\left\|T_{1}(\lambda)-T_{2}(\mu)\right\|_{\infty}=\| S_{1}(\lambda)-S_{2}(\mu)\right) \|_{\infty} .
$$

Then there exist a subset $Y_{0}$ of $Y$, a function $p \in B$ and continuous functions $\Phi: Y_{0} \rightarrow \Theta(A)$, $\alpha: Y_{0} \rightarrow[-1,1]$ and $w: Y_{0} \rightarrow \mathbb{T}$ where $\Phi$ is surjective such that

$$
T_{k}(\lambda)(y)=p(y)+w(y) \cdot\left(\operatorname{Re}\left(S_{k}(\lambda)(\Phi(y))\right)+\alpha(y) i \operatorname{Im}\left(S_{k}(\Phi(y))\right) \quad(k=1,2)\right.
$$

for all $f \in A$ and $y \in Y_{0}$.

Proof. Let $\widetilde{T}_{1}: A \rightarrow B$ be defined by $\widetilde{T}_{1}(f)=T_{1}(\lambda)$ where $\lambda \in I$ is an arbitrary element with $S_{1}(\lambda)=f$. Since for $\lambda, \mu \in I$ with $S_{1}(\lambda)=S_{1}(\mu)=f$, the norm condition (1) implies that $S_{2}\left(\mu^{\prime}\right)=S_{1}(\mu)$ where $\mu^{\prime} \in I$ satisfies $T_{2}\left(\mu^{\prime}\right)=T_{1}(\mu)$ it follows easily that $\widetilde{T}_{1}$ is well-defined. It is also easy to see that for $f, g \in A$

$$
\left\|\widetilde{T}_{1}(f)-\widetilde{T}_{1}(g)\right\|_{\infty}=\|f-g\|_{\infty}
$$

that is, $\widetilde{T}_{1}$ is an isometry. Thus by the Mazur-Ulam Theorem $\widetilde{T}_{1}-\widetilde{T}_{1}(0)$ is real-linear. Hence by Theorem 3.5 there exist a subset $Y_{0}$ of $Y$ and continuous functions $\Phi: Y_{0} \rightarrow \Theta(A)$, $\alpha: Y_{0} \rightarrow[-1,1]$ and $w: Y_{0} \rightarrow \mathbb{T}$, where $\Phi$ is surjective, such that

$$
\widetilde{T}_{1}(f)(y)-\widetilde{T}_{1}(0)(y)=w(y) \cdot(\operatorname{Re}(f(\Phi(y)))+\alpha(y) i \operatorname{Im}(f(\Phi(y))) .
$$


Setting $p=\widetilde{T}_{1}(0)$ we get

$$
T_{1}(\lambda)(y)-p(y)=w(y) \cdot\left(\operatorname{Re}\left(S_{1}(\lambda)(\Phi(y))\right)+\alpha(y) i \operatorname{Im}\left(S_{1}(\lambda)(\Phi(y))\right)\right.
$$

for all $\lambda \in I$ and $y \in Y_{0}$.

Now let $\widetilde{T}_{2}: A \rightarrow B$ be the isometry defined by $\widetilde{T}_{2}(f)=T_{2}(\mu)$ where for $f \in A$, $\mu$ is an element of $I$ satisfying $S_{2}(\mu)=f$. Then the norm condition (1) easily implies that $\widetilde{T}_{1}(f)=\widetilde{T}_{2}(f)$ for all $f \in A$. Hence (2) holds for $T_{2}$ and $S_{2}$ instead of $T_{1}$ and $S_{1}$, respectively, as desired.

ACKNOWLEDGEMENT. The authors would like to thank the referee for valuable suggestions and comments to improve the manuscript.

\section{References}

[ 1 ] J. Araujo and J. J. Font, Linear isometries between subspaces of continuous functions, Trans. Amer. Math. Soc. 349 (1997), 413-428.

[2] D. BLECHER and K. JAROSZ, Isomorphisms of function modules and generalised approximation in modules, Trans. Amer. Math. Soc. 354 (2002), 3663-3701.

[ 3 ] B. Cengiz, On extremely regular function spaces, Pacific J. Math. 49 (1973), 335-338.

[4] N. Dunford and J. Schwartz, Linear Operators, Part I, General Theory, Interscience Publishers Inc., New York, 1958

[ 5 ] A. J. ELLIS, Real characterizations of function algebras amongst function spaces, Bull. London Math. Soc. 22 (1990), 381-385.

[ 6] O. Hatori, G. Hiras awa and T. MiURA, Additively spectral-radius preserving surjections between unital semisimple commutative Banach algebras, Cent. Eur. J. Math. 8 (2010), 597-601.

[ 7 ] A. JAMSHIDI and F. SADY, Real-linear isometries between certain subspaces of continuous functions, Cent. Eur. J. Math. 11 (2013), 2034-2043.

[ 8 ] H. Koshimizu, T. Miura, H. TAKagi and S.-E. TAKahasi, Real-linear isometries between subspaces of continuous functions, J. Math. Anal. Appl. 413 (2014), 229-241.

[ 9 ] K. DE LeEuW, W. Rudin and J. Wermer, The isometries of some function spaces, Proc. Amer. Math. Soc. 11 (1960), 694-698.

[10] G. M. Leibowitz, Lectures on Complex Function Algebras, Scott, Foresman and Co., Glenview, Ill., 1970.

[11] T. MiURA, Real-linear isometries between function algebras, Cent. Eur. J. Math. 9 (2011), 778-788.

[12] W. Novinger, Linear isometries of subspaces of continuous functions, Studia Math. 53 (1975), 273-276.

[13] R. R. PhElPS, Lectures on Choquet's Theorem, 2nd Ed., Lecture Notes in Mathematics, 1757, Springer, 2001.

[14] N. V. RAO and A. K. RoY, Multiplicatively spectrum-preserving maps of function algebras II, Proc. Amer. Math. Soc. 133 (2004), 1135-1142.

[15] A. E. TAYlor and D. C. LAY, Introduction to Functional Analysis, 2nd Ed., Wiley, New York, 1980.

[16] T. ToneV and R. YATES, Norm-linear and Norm-additive operators between uniform algebras, J. Math. Anal. Appl. 57 (2009), 45-53. 
Present Addresses:

ARYA JAMSHIDI

Department of Pure Mathematics, Faculty of Mathematical Sciences,

TARBIAT MODARES UNIVERSITY,

TEHRAN, 14115-134, IRAN.

e-mail: Arya.Jamshidi@modares.ac.ir

FERESHTEH SADY

Department of Pure Mathematics, Faculty of Mathematical Sciences,

TARBIAT MODARES UNIVERSITY,

TEHRAN, 14115-134, IRAN.

e-mail: sady@modares.ac.ir 